\title{
Hypokalemic paralysis following administration of intravenous methylprednisolone in a patient with Graves' thyrotoxicosis and ophthalmopathy
}

\author{
Stelios Tigas, Petros Papachilleos, Nikolaos Ligkros, Maria Andrikoula, \\ Agathocles Tsatsoulis
}

Department of Endocrinology, University of Ioannina, Greece

\begin{abstract}
Glucocorticoids are commonly used in the treatment of patients with thyroid disorders, in particular Graves' ophthalmopathy. Thyrotoxic hypokalemic periodic paralysis (TPP) is an infrequent but potentially serious condition characterised by recurrent episodes of weakness associated with hypokalemia. We describe the development of acute hypokalemic paralysis in a middle-aged Caucasian man with recently diagnosed thyrotoxicosis and severe, active Graves' opthalmopathy who developed progressive flaccid paralysis 12 hours following intravenous administration of methylprednisolone. Rechallenge with the same dose after the patient had been rendered euthyroid did not provoke TPP. Clinicians should exercise caution when administering high-dose glucocorticoids during thyrotoxicosis as there is a risk of provoking hypokalemic paralysis in susceptible patients.
\end{abstract}

Key words: Glucocorticoids, Graves' disease, Graves' ophthalmopathy, Hypokalemia, Thyrotoxic hypokalemic periodic paralysis

\section{INTRODUCTION}

Glucocorticoids are used in clinical practice to treat patients with thyroid disorders; in addition to their anti-inflammatory and immunosuppressive properties, they inhibit peripheral conversion of T4 to T3, suppress TSH and reduce thyroid hormone

Address for correspondence:

Stelios Tigas, MD, PhD, MRCP, Dept of Endocrinology,

University of Ioannina, Ioannina 45110, Greece

Tel.: +30 26510 08089, Fax: +3026510 08098,

E-mail: stigas@cc.uoi.gr

Received 07-01-11, Revised 01-02-11, Accepted 28-02-11 secretion. ${ }^{1}$ Indications for their use include active moderate-to-severe Graves' ophthalmopathy (GO), dysthyroid optic neuropathy, amiodarone-induced destructive thyroiditis, subacute (de Quervain's) thyroiditis, prevention of deterioration of GO in certain radioiodine-treated patients and thyroid storm. ${ }^{2,3}$

Thyrotoxic hypokalemic periodic paralysis (TPP) is an infrequent but potentially serious condition characterised by recurrent episodes of weakness ranging from mild proximal muscle weakness to flaccid paralysis and associated with hypokalemia. ${ }^{4}$ The clinical presentation resembles that of familial hypokalemic periodic paralysis (FHPP), a frequently inherited 
disorder caused by a defect in muscle ion channels. ${ }^{5}$ We describe the development of acute hypokalemic paralysis in a patient with thyrotoxicosis following intravenous glucocorticoid administration for GO.

\section{CASE REPORT}

A 42 year-old man of Caucasian origin with recently diagnosed GO was referred to our outpatient clinic for evaluation and treatment. He had recently noticed a change in the appearance of his eyes and he complained of eye discomfort. In addition, he reported that in the preceding six months he had lost more than $15 \mathrm{~kg}$ in weight, without loss of appetite. He was a smoker. His past medical history was unremarkable, apart from alopecia universalis since childhood. On examination he appeared restless; he was obese with a body mass index of $34 \mathrm{~kg} / \mathrm{m}^{2}$. Body temperature was normal and there was a resting tachycardia of 100 beats/min. Blood pressure was 140/80 mm Hg. The skin was observed to be moist and warm and there was fine tremor of the outstretched hands. There were clinical signs of active GO: bilateral exophthalmos (32 $\mathrm{mm}$ on the right and $31 \mathrm{~mm}$ on the left, as measured by the Hertel exophthalmometer), lid aperture was 1 $\mathrm{cm}$ bilaterally and there was swelling and erythema of the eyelids and redness of the conjunctivae as well as corneal involvement (exposure keratopathy). The eye movements were restricted, particularly in upward gaze. The thyroid gland was diffusely enlarged and non-tender and there was no bruit on auscultation.

Biochemical testing revealed hyperthyroidism: total T4 was $288,3 \mathrm{nmol} / \mathrm{L}$ (NV 57.9-155.4) and total $\mathrm{T} 3$ was $>12.3 \mathrm{nmol} / \mathrm{L}$ (NV 0.8-2.1), accompanied by undetectable TSH $<0.01 \mathrm{mU} / \mathrm{L}$ (NV 0.5-4.8), elevated anti-thyroid peroxidase $(67 \mathrm{IU} / \mathrm{ml}$, normal $<35)$ and normal anti-thyroglobulin antibody level. Serum urea, creatinine, calcium, phosphate, liver transaminases and creatine kinase were normal. Serum potassium was $4.92 \mathrm{mEq} / \mathrm{L}$ (NV 3.5-5.3), sodium $142 \mathrm{mEq} / \mathrm{L}$ (NV 135-152), glucose $5.8 \mathrm{mmol} / \mathrm{L}, \mathrm{HbA} 1 \mathrm{c} 6.1 \%$ (4.6-6.2). The patient was started on high dose methimazole (40 mg daily in divided doses) and atenolol $50 \mathrm{mg}$ once daily. Computed tomography (CT) of the orbits revealed significant bilateral proptosis and extraocular muscle enlargement. Two days later he was admitted to the hospital for intravenous glucocorticoid pulse therapy. It turned out that his compliance had been poor; oral medication was given under supervision and the following day intravenous methylprednisolone was administered at a dose of $1 \mathrm{~g}$ in $500 \mathrm{ml}$ of normal saline over 3 hours. Twelve hours later the patient developed progressive flaccid paralysis with diminished tendon reflexes in the lower limbs, while the rest of the clinical examination was unremarkable. The serum potassium was $2.6 \mathrm{mEq} / \mathrm{L}$, with sodium, urea and creatinine being normal. The creatine kinase was $495 \mathrm{IU} / \mathrm{L}$ (40-190). At that time his plasma glucose was $196 \mathrm{mg} / \mathrm{dl}$. Electrocardiography revealed no abnormalities. The patient made a full recovery within hours following correction of the hypokalemia via intravenous potassium administration.

One month later he was readmitted to hospital for a second dose of intravenous glucocorticoids at a time when he had been rendered clinically and biochemically euthyroid: TSH was still undetectable but FT4 was 15.3 pmol/L (NV 9.0-23.8) and T3 was 1.92 $\mathrm{nmol} / \mathrm{L}$ (NV 0.8-2.1). Serum potassium on admission was $4.96 \mathrm{mmol} / \mathrm{L}$. After obtaining the patient's consent, the same dose $(1 \mathrm{~g})$ of methylprednisolone was administered under close monitoring of the serum potassium; the treatment was well tolerated this time without any weakness. Serum potassium remained normal at 4.71 and $4.42 \mathrm{mmol} / \mathrm{L}, 4$ and 12 hours after steroid administration, respectively.

\section{DISCUSSION}

Our patient developed hypokalemic thyrotoxic paralysis after a single dose of intravenous methylprednisolone. No hypokalemia or muscle weakness developed when exactly the same dose was administered one month later, at a time when the patient had been rendered euthyroid. It is plausible that glucocorticoid administration in the thyrotoxic phase triggered the development of TPP in this case.

Only a few cases of glucocorticoid-induced TPP have been reported in the literature. ${ }^{6-9}$ It has recently been determined that glucocorticoids may trigger attacks in several types of periodic paralysis. ${ }^{10}$ Although hypokalemia due to kaliuresis is a recognised sideeffect of glucocorticoid treatment, severe symptomatic glucocorticoid-induced hypokalemia is uncommon in routine clinical practice. ${ }^{11}$ Intravenous glucocorticoid 
administration for GO has been shown to be better tolerated and more effective compared to oral therapy and is currently standard practice. ${ }^{2,3}$

TPP typically affects young Asian men (incidence $\sim 2 \%$ in China and Japan among thyrotoxic patients) but is increasingly seen in non-Asian populations. ${ }^{4}$ Attacks usually involve the proximal muscles of the lower limbs in a symmetrical fashion and may progress to flaccid quadriplegia. Serious arrhythmias (sinus arrest, ventricular fibrillation) may occur. TPP attacks are more common in the summer and during the early hours of the morning and may be precipitated by consumption of carbohydrate-rich meals, alcohol or insulin administration. Strenuous exercise is another triggering factor, the hypokalemic paralysis typically occurring in the resting period following exercise. Non-selective $\beta$-blockers are thought to protect from TPP attacks by limiting $\mathrm{Na} / \mathrm{K}$-ATPase pump activation by the hyperadrenergic state associated with hyperthyroidism. In addition to the raised thyroid hormone levels, it is of note that prior to the attack our patient had developed glucocorticoid-induced hyperglycaemia and presumably hyperinsulinaemia, another potential contributing factor. ${ }^{12}$ Although urine potassium was not measured, the fact that (a) administration of exactly the same dose of methylprednisolone resulted in TPP during the hyperthyroid phase but not during euthyroidism, (b) the rapid rate of development and correction of the hypokalemia and (c) the absence of symptoms suggesting potassium loss in the gastrointestinal tract, would favor a shift of serum potassium into the cells as the cause of the hypokalemia.

The hypokalemia in TPP is postulated to result from increased $\mathrm{Na} / \mathrm{K}$-ATPase pump activity mainly in muscle cells, causing a rapid shift of potassium from the extracellular into the intracellular compartment. ${ }^{4,13}$ Factors eliciting acute stimulation of $\mathrm{Na} / \mathrm{K}$-ATPase pumps include catecholamines, $\beta_{2}$ adrenergic stimulation, insulin, IGF-1 and amylin; these effects are mediated via second messengers. ${ }^{14}$ Thyroid hormones and glucocorticoids appear to exhibit stimulatory effects by a different mechanism; they influence the content of $\mathrm{Na} / \mathrm{K}$-ATPase pumps by modifying their synthesis or degradation, an effect that requires more time. ${ }^{14}$ Ryan et al have recently reported that one in three patients with TPP carries a mutation of a gene encoding an inwardly rectifying potassium (Kir) channel, Kir2.6, suggesting that TPP might be a channelopathy like FHPP. ${ }^{15}$ Thus, it appears that during thyrotoxicosis the $\mathrm{Na} / \mathrm{K}$-ATPase pump content is upregulated in the skeletal muscles in addition to increased activity due to adrenergic stimulation; administration of glucocorticoids under these circumstances may provoke TPP, perhaps via hyperglycaemia and hyperinsulinaemia.

Although an infrequent complication of thyrotoxicosis, it is important that practising physicians are aware of the risk of provoking thyrotoxic paralysis when using high-dose glucocorticoids in the thyrotoxic phase, particularly since intravenous steroid use for Graves' orbitopathy is being increasingly applied and TPP, if anticipated, could be prevented or alleviated. Moreover, the use of nonselective $\beta$-blockers may also prevent or ameliorate TPP. ${ }^{4}$ Close monitoring of serum potassium is mandatory and, if needed, potassium should be supplemented at a slow rate to prevent rebound hyperkalemia during recovery of the paralysis. ${ }^{4}$ Alternatively, and if the patient's clinical condition permits (e.g. in the absence of dysthyroid optic neuropathy), it may be preferable to defer the use of glucocorticoids for a few weeks until the patient is rendered euthyroid.

In conclusion, clinicians should exercise caution when administering glucocorticoids during thyrotoxicosis as there is a risk of provoking hypokalemic paralysis in susceptible individuals.

\section{REFERENCES}

1. Surks MI, Sievert R, 1995 Drugs and thyroid function. N Engl J Med 333: 1688-1694.

2. Bartalena L, Baldeschi L, Dickinson AJ, et al, 2008 Consensus statement of the European group on Graves' orbitopathy (EUGOGO) on management of Graves' orbitopathy. Thyroid 18: 333-346.

3. Perros P, Neoh C, Dickinson J, 2009 Thyroid eye disease. BMJ 338: 645-650.

4. Kung AW, 2006 Clinical review: Thyrotoxic periodic paralysis: a diagnostic challenge. J Clin Endocrinol Metab 91: 2490-2495.

5. Finsterer J, 2008 Primary periodic paralyses. Acta Neurol Scand 117: 145-158.

6. Wessel K, Schumm F, Peiffer J, Schlote W, 1985 Hypokalemic periodic paralysis provoked by "Ambene". Nervenarzt 56: 696-702. 
7. Miyashita Y, Monden T, Yamamoto K, et al, 2006 Ventricular fibrillation due to severe hypokalemia induced by steroid treatment in a patient with thyrotoxic periodic paralysis. Intern Med 45: 11-13.

8. Liu Z, Braverman LE, Malabanan A, 2006 Thyrotoxic periodic paralysis in a Hispanic man after the administration of prednisone. Endocr Pract 12: 427-431.

9. Wongraoprasert S, Buranasupkajorn P, Sridama V, Snabboon T, 2009 Thyrotoxic periodic paralysis induced by pulse methylprednisolone. Intern Med 46: 1431-1433.

10. Arzel-Hézodea M, McGoeybc S, Sternbergabc D, Vicartabc S, Eymarda B, Fontaine B, 2009 Glucocorticoids may trigger attacks in several types of periodic paralysis. Neuromuscular Disorders 19: 217-219.

11. Tamez-Pérez HE, Cisneros-Pérez V, Cedillo-Rodríguez JA, et al, 2009 Prevalence of hypokalemia in patients with methylprednisolone pulse therapy. Rev Invest Clin 61: 194-197.

12. Chan A, Shinde R, Chow CC, Cockram CS, Swaminathan $\mathrm{R}, 1994$ Hyperinsulinaemia and $\mathrm{Na}+, \mathrm{K}(+)$-ATPase activity in thyrotoxic periodic paralysis. Clin Endocrinol (Oxf) 41: 213-216.

13. Chan A, Shinde R, Chow CC, Cockram CS, Swaminathan $\mathrm{R}, 1991$ In vivo and in vitro sodium pump activity in subjects with thyrotoxic periodic paralysis. BMJ 303: 1096-1099.

14. Clausen T, $2003 \mathrm{Na}^{+}-\mathrm{K}^{+}$pump regulation and skeletal muscle contractility. Physiol Rev 83: 1269-1324.

15. Ryan DP, Dias da Silva MR, Soong TW, 2010 Mutations in Potassium Channel Kir2.6 Cause Susceptibility to Thyrotoxic Hypokalemic Periodic Paralysis. Cell 140: 88-98. 